\section{A simplified assay can predict superior outcome in patients with B-lineage ALL}

Treatment of pediatric acute lymphoblastic leukemia (ALL) is most effective when the risk of relapse is assessed early; the response of leukemia cells to treatment reliably predicts prognosis. Assays have been developed that can measure tiny amounts of leukemia, or 'minimal residual disease' (MRD), but their cost and complexity has slowed their universal application.

Coustan-Smith et al. present a simplified flow cytometric assay that detects immature CD19+ cells - those expressing CD10 and/or CD34-in patients with $B$-lineage ALL early in the course of remission induction therapy. Absence of these cells can indicate early treatment success.

Samples taken from 380 children with B-lineage ALL on day 19 of treatment were tested for MRD. Results from the simplified flow cytometric assay correlated closely with those from standard MRD assays. The simplified assay was also highly sensitive, uncovering residual disease in $50.6 \%$ of the 342 samples morphologically negative for leukemic lymphoblasts. Among 84 patients enrolled on an identical treatment regimen, the 5-year cumulative remission-failure or relapse rate for those in whom immature CD19+ cells were undetectable on day 19 was $4.8 \%$, compared with $23.8 \%$ for patients in whom immature CD19+ cells were detected. Successful use of the simplified flow cytometric assay in a laboratory in Recife, Brazil, demonstrated its suitability for use in resource-poor environments.

The authors suggest that the assay could facilitate the routine identification of patients with ALL whose disease is highly sensitive to chemotherapy and who are therefore suitable for lower intensity treatment.

Original article Coustan-Smith E et al. (2006) A simplified flow cytometric assay identifies children with acute lymphoblastic leukemia who have a superior clinical outcome. Blood 108: 97-102

\section{Histamine dihydrochloride and interleukin 2 protect against relapse in AML}

Most patients with acute myeloid leukemia (AML) achieve complete remission after induction chemotherapy, but many will eventually relapse; the long-term disease-free survival rate is $25-35 \%$. A multicenter, randomized phase III trial has shown that combination histamine dihydrochloride and interleukin 2 (HDC/IL-2) can protect against relapse, by activating cytotoxic lymphocytes to attack residual malignant cells.

In total, 320 patients (aged 18-84 years; median age 57 years) in remission from AML were recruited and randomly assigned to receive either 18 months of HDC/IL-2, or the current standard of care (i.e. no treatment). The rate of leukemia-free survival was significantly higher in patients who received HDC/IL-2 than in those who received no treatment $(P<0.01)$. For patients in first remission, the percentage remaining in remission after 3 years was $40 \%$ in the treated group compared with $26 \%$ in the control group. For patients in second or subsequent remission, however, HDC/IL-2 treatment brought no advantage; in all, only 7 of the 59 patients with previous relapse who participated in the trial were disease-free after 3 years.

It has been shown that inhibitory factors released by myeloid cells suppress the activity of cytotoxic lymphocytes. HDC targets these suppressive mechanisms, and the HDC/IL-2 combination has been shown in vitro to facilitate the destruction of leukemic cells, whereas either agent alone is ineffective. IL-2 has exhibited considerable toxicity in previous trials, but treatment was well tolerated at the much lower IL-2 dose used in this study. The results indicate that HDC/IL-2 is a safe and effective treatment for patients in first remission from AML.

Original article Brune M et al. (2006) Improved leukemiafree survival after postconsolidation immunotherapy with histamine dihydrochloride and interleukin-2 in acute myeloid leukemia: results of a randomized phase 3 trial. Blood 108: 88-96

\section{Increasing radiation dose affects risk classification for prostate cancer}

Classification of patients with prostate cancer into risk groups has been based on patient characteristics such as pretreatment prostatespecific antigen levels and Gleason score; however, it is possible that patient-related risk factors are not independent of treatment-related factors. As increasingly higher doses are being used, Thames et al. hypothesized that changes 